\title{
ORIGINAL ARTICLE Enhancing the cytotoxicity of chemoradiation with radiation-guided delivery of anti-MGMT morpholino oligonucleotides in non-methylated solid tumors
}

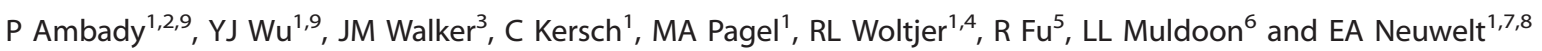

The DNA repair enzyme $0^{6}$-methylguanine DNA methyltransferase (MGMT) is epigenetically silenced in some tumors by MGMT gene promoter methylation. MGMT-hypermethylated solid tumors have enhanced susceptibility to the cytotoxic effects of alkylating chemotherapy such as temozolomide, compared with non-methylated tumors. In glioblastoma, subjects with MGMT hypermethylation have significantly longer survival rates after chemoradiotherapy. We report the first successful use of a non-ablative dose of ionizing radiation to prime human cancer cells to enhance the uptake of unmodified anti-MGMT morpholino oligonucleotide (AMON) sequences. We demonstrate $>40 \%$ reduction in the in vitro proliferation index and cell viability in radiation-primed MGMT-expressing human solid tumor cells treated with a single dose of AMONs and temozolomide. We further demonstrate the feasibility of using a non-ablative dose of radiation in vivo to guide and enhance the delivery of intravenously administered AMONs to achieve 50\% MGMT knockdown only at radiation-primed tumor sites in a subcutaneous tumor model. Local upregulation of physiological endocytosis after radiation may have a role in radiation-guided uptake of AMONs. This approach holds direct translational significance in glioblastoma and brain metastases where radiation is part of the standard of care; our approach to silence MGMT could overcome the significant problem of MGMT-mediated chemoresistance.

Cancer Gene Therapy (2017) 24, 348-357; doi:10.1038/cgt.2017.27; published online 28 July 2017

\section{INTRODUCTION}

Glioblastoma is an aggressive primary brain tumor that carries a poor prognosis even after aggressive resection and standard-ofcare chemoradiotherapy (CRT). The standard regimen includes 60 Gy radiation delivered over 6 weeks in 1.8-2 Gy dose fractions per day concurrently with the oral alkylating chemotherapy temozolomide; this is followed by at least 6 additional months of adjuvant cycles of temozolomide. ${ }^{1}$ The cytotoxic effects of temozolomide in this setting are mediated by methylation of $\mathrm{O}^{6}$ and $N^{7}$ positions of guanylic acid and the $N^{3}$ position of adenine resulting in a continuous cycle of DNA base mismatch with eventual DNA strand breaks that ultimately triggers apoptosis. The DNA repair enzyme $0^{6}$-methylguanine DNA methyltransferase (MGMT) is a suicide enzyme that unmethylates these sites to restore genome stability. ${ }^{2-8}$ Via this mechanism, MGMT enzyme expression has a key role in resistance to CRT. ${ }^{2,3,5,6}$ The MGMT gene is epigenetically silenced in about $45 \%$ of glioblastoma cases by methylation of CPG islands, predominantly located in the $5^{\prime}$ gene promoter region. ${ }^{9}$ Tumor cells with MGMT gene promoter methylation have enhanced susceptibility to the cytotoxic effects of radiation and/or alkylating agents such as temozolomide due to their inability to repair the CRT-mediated DNA damage. This correlates with a significant survival benefit in glioblastoma patients with MGMT promoter methylation who receive
CRT. ${ }^{2,8-10}$ Silencing MGMT is thus a promising therapeutic target in glioblastoma and other solid tumors such as lung, breast and renal cell carcinoma where this enzyme is known to have a role in chemoresistance. $^{11-13}$

Antisense morpholino oligonucleotides are short custom-built sequences of uncharged nucleic acid analogs that are composed of nitrogen base pairs bound to morpholino rings, instead of ribose or deoxyribose rings, linked through uncharged phosphorodiamidate groups instead of anionic phosphates. ${ }^{14,15}$ They have emerged as excellent tools in vitro for blocking sites on RNA to prevent translation and thereby inhibit protein expression. We evaluated the use of novel anti-MGMT oligonucleotides (AMONs) developed on a neutral morpholino backbone that can effectively silence MGMT both in vitro and in tumor models.

The target specificity of morpholino oligomers and their stability in vivo makes them excellent candidates for potential therapeutic applications in humans. ${ }^{14-17}$ However, intracellular delivery, especially into the brain across the blood-brain barrier (BBB), remains a major challenge and limits clinical translation for brain tumor therapy. ${ }^{18-20}$ Internalization of antisense oligomers is at least partially mediated by endocytosis. ${ }^{21-23}$ lonizing radiation is known to enhance endocytosis. ${ }^{24}$ As RT is already an integral part of 'standard-of-care' treatment for primary and metastatic brain tumors, we proposed this as a method to potentially enhance

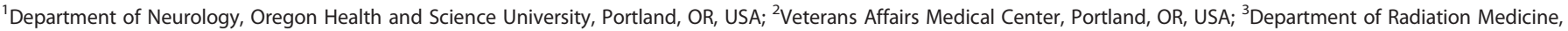

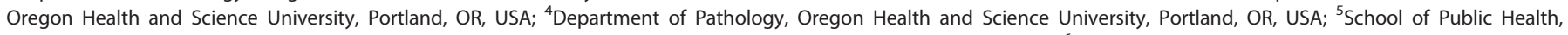

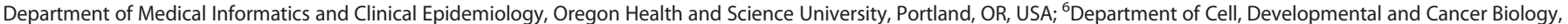

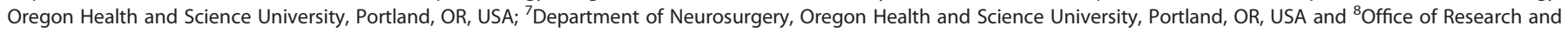

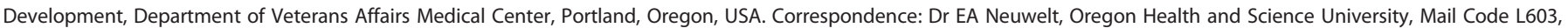
3181 SW., Sam Jackson Park Road, Portland, OR 97239, USA.

E-mail: neuwelte@ohsu.edu

${ }^{9}$ These authors contributed equally to this manuscript.

Received 4 May 2017; accepted 20 May 2017; published online 28 July 2017 
cellular uptake of morpholinos. ${ }^{1,25}$ In this report, we demonstrate the efficacy of a non-lethal dose of ionizing radiation to guide and enhance the intracellular uptake of unmodified antisense morpholino oligonucleotide sequences and to silence MGMT protein expression.

\section{MATERIALS AND METHODS}

Cell lines

Human T98G glioma, H460 and A549 non-small cell lung carcinoma (NSCLC) cell lines were obtained from American Type Culture Collection (ATCC; Rockville, MD, USA) and cultured in recommended culture medium supplemented with in $10 \%$ fetal bovine serum and 1\% streptomycin/ penicillin. Cells were maintained in an atmosphere containing $5 \% \mathrm{CO}_{2}$ at $37^{\circ} \mathrm{C}$.

\section{Reagents}

AMONs: Three AMON sequences and control sequences (Table 1) were synthesized and purchased from Gene Tools, LLC (Philomath, OR, USA). Temozolomide for injection was purchased Merck \& Co., Inc. (Whitehouse Station, NJ, USA) and diluted in sterile water to a concentration of $2.5 \mathrm{mg} \mathrm{ml}^{-1}$. Mouse anti-tubulin antibody (T9026) was purchased from Sigma (St Louis, MO, USA). In western blotting studies, anti-MGMT (\#2739), caspase 3 (\#9662), caveolin1 (\#3267), rab5 (\#3547), clathrin (\#4796), EEA1 (\#3288), APPL1 (\#3858) and phospho-p53 (Ser15) (\#9284) antibodies were purchased from Cell Signaling Technology (Danvers, MA, USA). Mouse antidynamin 2 (sc-166526) and p27 (sc-527) antibodies were purchased from Santa Cruz Biotech. (Santa Cruz, CA, USA). For immunohistochemistry, Rabbit anti-MGMT (171195-1-AP) and ki67 (sc-15402) antibodies were purchased from Proteintech (Rosemont, IL, USA) and Santa Cruz Biotech, respectively.

\section{AMON delivery techniques}

Scrape loading. Cell were placed on a flat surface and gently scraped off the surface with a sterile cell scraper (Sarstedt, Inc., Newton, NC, USA), according to the manufacturer's protocol. ${ }^{26}$ Different doses or combinations of MGMT morpholinos were added; scraped cell suspension was then gently pipetted up and down twice and transferred to another culture plate.

Transfection agents. Cells were treated with transfection agent DharmaFECT (GE Dharmacon, Lafayette, CO, USA) and MGMT-morpholinos mixture $(1: 1 ; \mathrm{v} / \mathrm{v})$ without scraping the cells according to the manufacturer's protocol. ${ }^{26}$

Radiation-guided delivery. A total of $10^{6}$ cancer cells (T98G, H460 and A549) were exposed to different doses of radiation (0.5-12 Gy) at room temperature using a Philips gamma chamber (Philips Medical Systems, Andover, MA, USA). After irradiation, cells were treated with mixtures of MGMT morpholinos (\#1:\#2:\#3=1:1:1; v/v/v) at different time points and at the final concentration of $15 \mu \mathrm{m}$. For the purpose of this study, non-ablative radiation is defined as radiation doses $<8 \mathrm{~Gy}$ per fraction. ${ }^{27}$ Cells were

Table 1. Summary of oligonucleotide sequences used in this paper

\begin{tabular}{lllll}
\hline Morpholinos & Nucleotide sequence $5^{\prime} \rightarrow 3^{\prime}$ & $\begin{array}{c}\text { No. of } \\
b p^{\text {a }}\end{array}$ & MW \\
\hline Anti-MGMT specific & 21 & 7037 \\
Sequence \#1 & 5'-TTTCGTGCAGACCCTGCTCTT-3' & 21 & 7045 \\
Sequence \#2 & 5'-TTCCATAACACCTGTCTGGTT-3' & 21 & 7006 \\
Sequence \#3 & $5^{\prime}$-ATTCCTTCACGGCCAGTCCTT-3' & & \\
Nonspecific & 5'-CCTCTTACCTCAGTTACAATTTATA-3' & 25 & 8328 \\
Fluorescent & 5'-CCTCTTACCTCAGTTACAATTTATA & 25 & 8817 \\
nonspecific & -carboxyfluorescein-3' & & \\
\hline
\end{tabular}

Abbreviations: A, adenosine; C, cytosine; G, guanine; MGMT, $\mathrm{O}^{6}$-methylguanine DNA methyltransferase; MW, molecular weight; $\mathrm{T}$, thymine. ${ }^{a}$ Number of base pair in nucleotides. radiated with different doses of radiation (0.5-12 Gy). Mixtures of AMONs (\#1:\#2:\#3=1:1:1; $5 \mu \mathrm{m}$ each) or no drug controls were added 1 day after radiation, and then temozolomide $\left(150 \mu \mathrm{g} \mathrm{ml}^{-1}\right.$, final concentration) was added into selected wells 2 days after AMONs. Cell viability was determined at specific time (1-4 days) after temozolomide treatment using WST-1 reagent (Abcam, Cambridge, MA, USA), following the manufacturer's protocol.

Cell viability assay and western blotting analyses

For western blotting analysis, cells (T98 G or H460 cell lines)) or tissues (H460 cells) were harvested and then washed with ice-cold phosphatebuffered saline (PBS) twice before the addition of RIPA lysis buffer containing protease and phosphatase inhibitors (Roche, Indianapolis, IN, USA). Protein concentration was quantified using the bicinchoninic acid protein assay. Equal amounts of protein ( $20 \mu \mathrm{g}$ per lane) were loaded into each well and separated by 7.5 or $12 \%$ sodium dodecyl sulfatepolyacrylamide gel electrophoresis followed by transfer onto polyvinylidene difluoride membranes (Bio-Rad, Richmond, CA, USA). Membranes were blocked using $5 \%$ nonfat dry milk or $2 \%$ bovine serum albumin (in PBS with $0.05 \%$ tween-20. The blots were then incubated with primary antibodies $(1: 200-1: 1000)$ overnight at $4{ }^{\circ} \mathrm{C}$. Secondary antibody anti-rabbit or anti-mouse IgG conjugated to horse radish peroxidase (1:5000; Cell Signaling Technology) was incubated for $2 \mathrm{~h}$ at room temperature. Immunoblots were developed using the chemiluminescence detection system (Thermo Scientific, Waltham, MA, USA). Quantification of immunoblotting signal of respective proteins was performed using the UN-SCAN-IT Gel software (Silk Scientific, Inc., Orem, UT, USA).

\section{Immunohistochemistry}

Cells were exposed to radiation ( $1 \mathrm{~Gy}$ ) or control no radiation 1 day after plating; $24 \mathrm{~h}$ after radiation, cells were incubated with mixtures of AMONs (\#1:\#2:\#3=1:1:1; $5 \mu \mathrm{m}$ each); and temozolomide $\left(150 \mu \mathrm{g} \mathrm{ml}^{-1}\right.$, final concentration) was added into selected wells 2 days after AMONs. Cells were fixed in fresh $4 \%$ paraformaldehyde and permeabilized with $0.1 \%$ Triton $X$ at room temperature for $15 \mathrm{~min}$ and then washed with PBS. Immunohistochemistry that was performed followed the procedure as described previously. ${ }^{28}$ Representative images are shown; all images are optical sections. All images were collected using identical settings on the microscope. MGMT was considered positive when uniform nuclear MGMT staining was detected, while immunostaining in the cytoplasmic and granular nuclear reactivity was considered negative as previously described ${ }^{29,30}$

\section{Confocal microscopy}

T98G cells were exposed to radiation (1 Gy) or controls no radiation; either $24 \mathrm{~h}$ after radiation $(1 \mathrm{~Gy})$ or no radiation, cells were incubated with GFP-morpholino (1:500) and Texas red conjugated WGA (1:500) for $1 \mathrm{~h}$ at $37^{\circ} \mathrm{C}$ and then fixed in fresh $3.7 \%$ paraformaldehyde at room temperature for $15 \mathrm{~min}$ and then washed with PBS. Membranes were not permeabilized. Images were collected the following day using an 60x/1.4 NA oil immersion objective on an inverted Zeiss LSM780 confocal microscope maintained by the Oregon Health and Science University (OHSU) Advanced Light Microscopy Core (Carl Zeiss, Oberkochen, German); images were analyzed using FIJI/ImageJ (NIH, Bethesda, MD, USA, https://fiji.sc). ${ }^{31}$ Representative images are shown; all images are optical sections. All images were collected using identical settings on the microscope.

\section{Measurement of MGMT DNA methylation status}

DNA from subcutaneous xenograft tumors (xenograft method described in detail below) was isolated using the DNeasy Blood \& Tissue Kit from Qiagen International (Germantown, MD, USA). The percentage of methylation status of MGMT DNA at CpG islands in samples were measured at OHSU Knight Cancer Center Clinical Laboratories Service using pyrosequencing technique using Pyromark ID (Qiagen, Valencia, CA, USA). ${ }^{32,33}$

Subcutaneous tumor model and in vivo delivery of AMONs with non-ablative ionizing irradiation

The care and use of the animals was approved by the institutional animal care and use committee and was under the supervision of the Department of Comparative Medicine at OHSU. Female (200-250 g) nude ( $r n u / r n u)$ rats 
were selected from the breeding colony maintained in the animal facility at OHSU. For the subcutaneous tumor model, a total of $2.5 \times 10^{7}$ cells were mixed (ratio 1:1; v/v) with Matrigel Basement Membrane Matrix (BD Biosciences, Bedford, MA, USA), and the mixture was injected subcutaneously into each hind limb. At day 7 after inoculation, the right subcutaneous tumor was exposed to single computer tomography-guided priming dose of radiation (5 Gy) using a Versa HD (Elekta, Stockholm, Sweden) linear accelerator in the Department of Radiation Oncology, and left subcutaneous tumor of the same animal without radiation served as control. One centimeter of tissue equivalent bolus material was overlaid onto the RT field to ensure adequate dose to the most superficial portion of the lesion. A field size of $3 \times 5 \mathrm{~cm}^{2}$ was used and a half beam block technique was implemented in order to limit dose outside the RT field. Dose was confirmed using NanoDot (Landauer, Glenwood, IL, USA) optically stimulated luminescence dosimetry. In vivo animal experiments were performed in sets of two animals each where both received radiation to their right hind limb while only one animal received AMON, given intravenously (IV) as a total of $10.5 \mathrm{mg} \mathrm{kg}^{-1}$ mixtures of AMONs (\#1:\#2: $\# 3=1: 1: 1)$ through the femoral vein $24 \mathrm{~h}$ after radiation. Tumor tissues were harvested 3 days after AMONs were administered and subjected to protein and DNA analysis. This experiment was repeated independently three times $(n=6)$.

\section{Statistical analysis}

The results were expressed as mean \pm s.d. For percentage of cells with nuclear MGMT staining or ki67+ staining, one-way analysis of variance models were used to compare differences between the different treatment groups. When there were two treatment factors (RT; AMON/ TMZ), two-way analysis of variance models were used to compare relative p-p53 level, relative cell viability and relative MFMT level, where the relative values were obtained by dividing the raw data by the value from the control group. Multiple comparisons were adjusted using Tukey's correction. Significance was determined at the $5 \%$ level, two sided. Statistical significance between treatment and control (or vehicle) groups or any two other groups was indicated by ${ }^{*} P<0.05$, ${ }^{* *} P<0.01$ or ${ }^{* * *} P<0.001$.

\section{RESULTS}

lonizing radiation enhances AMON delivery to silence MGMT

Expression of MGMT was verified in multiple cancer cell types and three MGMT-expressing solid tumor cell lines (T98G glioma, and H460 and A549 NSCLC) were selected for further experiments. Three AMON sequences were tested to identify to be the best combination to facilitate MGMT silencing (Table 1). T98G cells were exposed to sequences 2, 3 or the combination of 1, 2 and 3, $1 \mathrm{~h}$ after exposure to $1 \mathrm{~Gy}$ of ionizing radiation. Western blottings performed on cell lysates obtained 3 days after exposure to the sequences demonstrate almost complete (100\%) silencing with the combination of sequences 1,2 and 3, (Figure 1a) compared with sequences 2 and 3 or sequence 3 alone. The knockdown achieved with radiation-enhanced delivery of AMONs was transient, with knockdown at 3 days and restoration of MGMT expression by 7 days (Figure 1a). The combination of sequences 1 , 2 and 3 (1:1:1 ratio) were used in future experiments in the current study unless specifically indicated. To determine the optimal timing for exposure to AMON with respect to exposure to radiation, T98G cells were exposed to AMONs at different time points, $2 \mathrm{~h}$ and $1 \mathrm{~h}$ before radiation as well as 1 and $24 \mathrm{~h}$ after radiation exposure. Of the 5 different time points we investigated, the best MGMT knockdown $(87.4 \pm 5.3 \%)$ was achieved when cells were exposed to AMON $24 \mathrm{~h}$ after radiation compared with nonradiated control (Figure $1 \mathrm{~b}$ ). No time points $>24 \mathrm{~h}$ after radiation were assessed. MGMT gene expression knockdown was confirmed with immunohistochemistry for MGMT protein (Figures $1 \mathrm{c}$ and d), which demonstrated a $43.2 \pm 6.5 \%$ reduction in nuclear MGMT staining in T98G cells exposed to AMONs $24 \mathrm{~h}$ after $1 \mathrm{~Gy}$ radiation. No significant reduction in nuclear MGMT was noted when cells were exposed to either radiation alone or when treated with AMON without radiation. The radiation dose needed to facilitate cellular delivery of AMONs varied between tumor cell lines. Knockdown could be achieved in T98G cell lines at $1 \mathrm{~Gy}$ while H460 and A549 cell lines required exposure to 6-12 Gy for best results with no-to-lesser degree of MGMT knockdown at lower doses of ionizing radiation (Figures $1 \mathrm{a}$, e and $\mathrm{f}$ ). Similar trends $(18.2 \pm 4.2 \%$ reduction in nuclear MGMT staining) were found in H460 cells exposed to AMONS $24 \mathrm{~h}$ after 6 Gy radiation (Figure $1 \mathrm{~g}$ ). Alternate delivery techniques including scrape delivery and liposomal transfection agents, two well-described methods for in vitro delivery of oligonucleotides into the cytosol of cultured cells, were additionally tested (Supplementary Figure 1). ${ }^{26} \mathrm{~A}$ combination of sequences 1,2 and $3(1: 1: 1)$ at a dose of $15 \mu \mathrm{m}$ resulted in $95 \pm 7.5 \%$ MGMT silencing at day 3 with scrape loading (Supplementary Figures $1 \mathrm{a}$ and b) or liposomal transfection agent $(47.3 \pm 8.5 \%$ knockdown; Supplementary Figure 1c) techniques; MGMT silencing was not achieved when cells were exposed to AMONs without radiation priming, scrape loading or liposomal transfection agents (controls).

\section{Silencing MGMT using AMONs enhances the cytotoxicity of temozolomide}

Once radiation-enhanced delivery of AMONs and MGMT silencing was confirmed, we tested whether this silencing could enhance the cytotoxicity of temozolomide. T98G cells were exposed to $1 \mathrm{~Gy}$ radiation (day 0 ), followed by AMONs (total of $15 \mu \mathrm{M}$; sequences 1 , 2 and 3 in 1:1:1 ratio) on day 1 (Figure 1a). Cells were then exposed to 0,75 or $150 \mathrm{\mu g} \mathrm{ml}^{-1}$ of temozolomide solution on day 4 , respectively. Lysates were collected on day 5 for immunoblotting assay. Our results suggest that cells that received AMONs followed by temozolomide showed a dose-dependent increase in early apoptosis (phosphorylated-p53 protein) at day 1 compared with those that received no AMONs (Figures $2 a$ and $b$ ). To confirm our findings, we performed WST-1 cell viability assay in T98G and H460 cells. Cells received $1 \mathrm{~Gy}$ of radiation on day 0 followed by either $150 \mu \mathrm{g} \mathrm{ml}^{-1}$ of temozolomide solution on day 3 or a combination of AMONs and temozolomide (AMONs on day 1 followed by temozolomide on day 3). Cells that received $1 \mathrm{~Gy}$ radiation only with no addition treatments served as controls. Cell viability was assessed on days $4-7$. Our results show a statistically significant decline in T98G cell viability in the group that received AMONs and temozolomide compared those that received temozolomide only (Figure 2c) and a similar trend in H460 NSCLC cells. Immunohistochemistry (Figures $2 \mathrm{~d}$ and e) demonstrates a statistically significant $(P<0.01)$ decrease in the number of proliferating T98G cells $(46.3 \pm 10.6 \%$ ki67+ cells) exposed to AMON and temozolomide compared with those that received temozolomide $24 \mathrm{~h}$ after $1 \mathrm{~Gy}$ radiation $(74.5 \pm 1.2 \%)$ or radiationonly controls $(82.7 \pm 11.2 \%)$.

Radiation-guided enhanced delivery of IV administered AMONs downregulate MGMT in a subcutaneous tumor model

Owing to inconsistent growth of T98G cells in vivo, MGMTexpressing $\mathrm{H} 460$ NSCLC subcutaneous tumors were used for our in vivo animal model. Six animals were subcutaneously injected with $2.5 \times 10^{7} \mathrm{H} 460$ cells in each of their hind limbs. When the tumors reached $10 \mathrm{~mm}$ in diameter (1 week after implantation), a single dose of $5 \mathrm{~Gy}$ of ionizing radiation was delivered only to the right hind limb tumor of each animal (Figure 3a). Half of the animals $(n=3)$ then received IV AMONs 1 day after radiation, and tumors were harvested 3 days after exposure to AMONs. We found a $50 \pm 9.7 \%$ reduction in MGMT expression by western blotting assay in tumors primed with radiation prior to IV administration of AMONs. MGMT knockdown was not seen in control tumors that either received IV AMONs without prior radiation or received radiation alone (Figures $3 \mathrm{~b}$ and $\mathrm{c}$ ). Dosimetry confirmed radiation dose to each tumor site. Further, elevated p27 expression was used as a biomarker to confirm radiation exposure to the right 
a

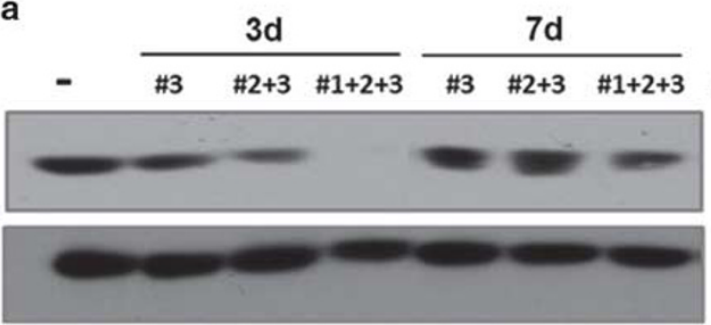

b

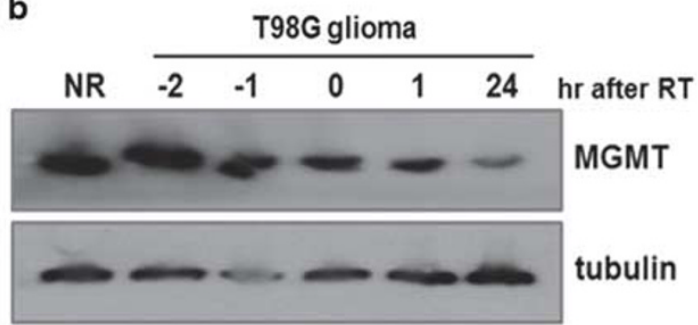

d

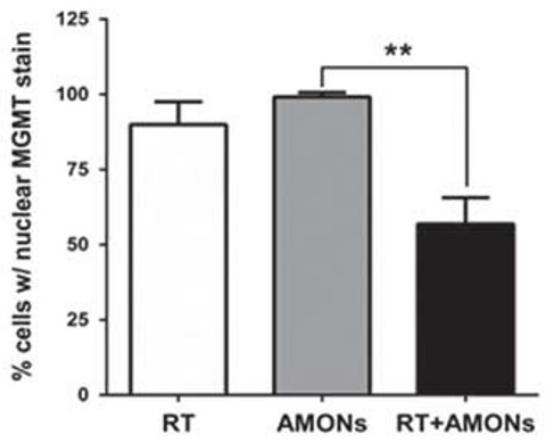

e
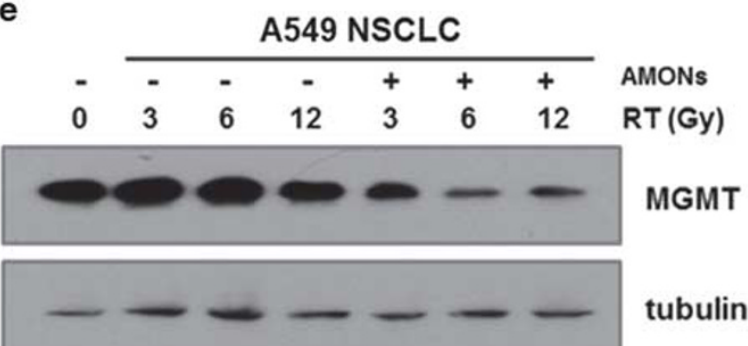

$<$

$<<$

$<$

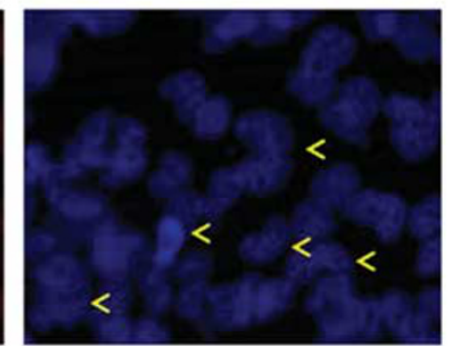

$\mathbf{f}$

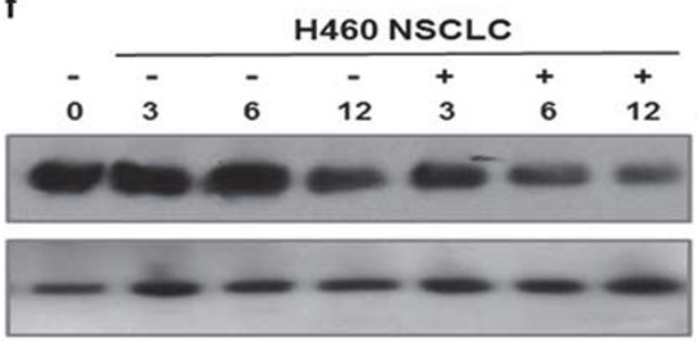

AMONs RT (Gy)

MGMT

tubulin

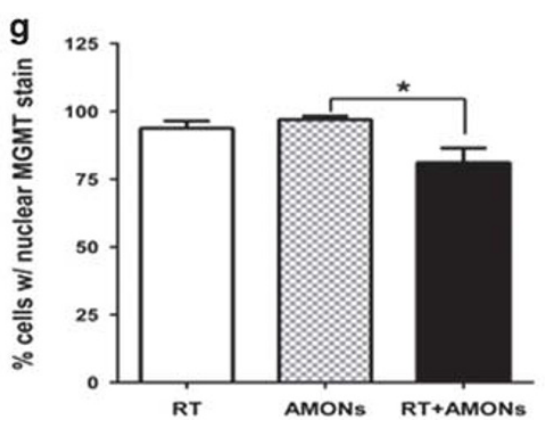

Figure 1. Radiation enhances anti-MGMT oligonucleotide (AMON) delivery and reduces $\mathrm{O}^{6}$-methylguanine DNA methyltransferase (MGMT) protein expression in human cancer cells. (a) Downregulation in MGMT expression after treatment with a single dose of AMONs (combination of sequences 1, 2 and 3 in 1:1:1 ratio; $5 \mu \mathrm{m}$ each) in radiation-primed T98G cells was seen at 3 days, and MGMT expression returned to pretreatment baseline by 7 days; (b) Immunoblot demostrates the time-dependent downregulation of MGMT expression in T98G glioma cells treated with AMONs after a priming dose of non-ablative dose of radiation (1 Gy), compared with nonradiated (NR) cells. Maximal reduction in MGMT expression was noted after combination of sequences 1, 2 and 3 (1:1:1 ratio; $5 \mu \mathrm{m}$ each were given $24 \mathrm{~h}$ after radiation); (c) Compared with cells treated with AMONs or radiation alone, radiation plus AMONs decreased the percentage of nuclear MGMT-positive-stained cells (indicated by arrowhead) in T98G cells; (d) Quantification of three random MGMT immunohistochemical fields shown in panel (c). Data are presented as mean \pm s.e.m.; (e, f) The dose of radiation required for optimal delivery of AMONs is tumor cell-type specific. Downregulation of MGMT by AMONs 3 days after AMONs in (e) A549 and (f) H460 non-small cell lung carcinoma (NSCLC) cells was in a radiation dose-dependent manner. RT, radiotherapy. (g) Quantification of 3 random MGMT IHC fields of H460 cells treated with RT, AMONs or RT+AMONs. 
a

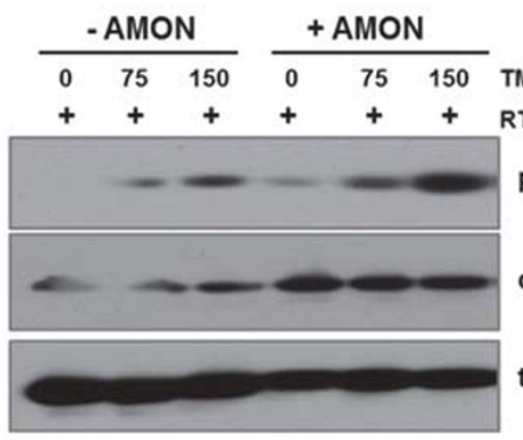

b

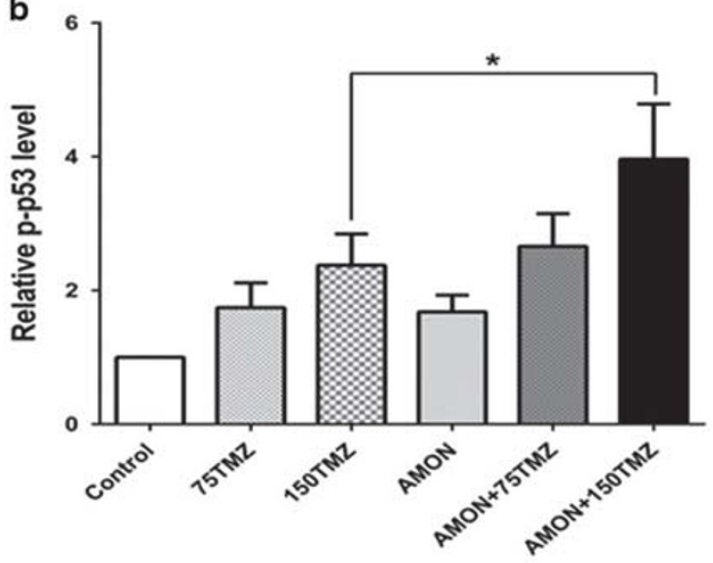

d
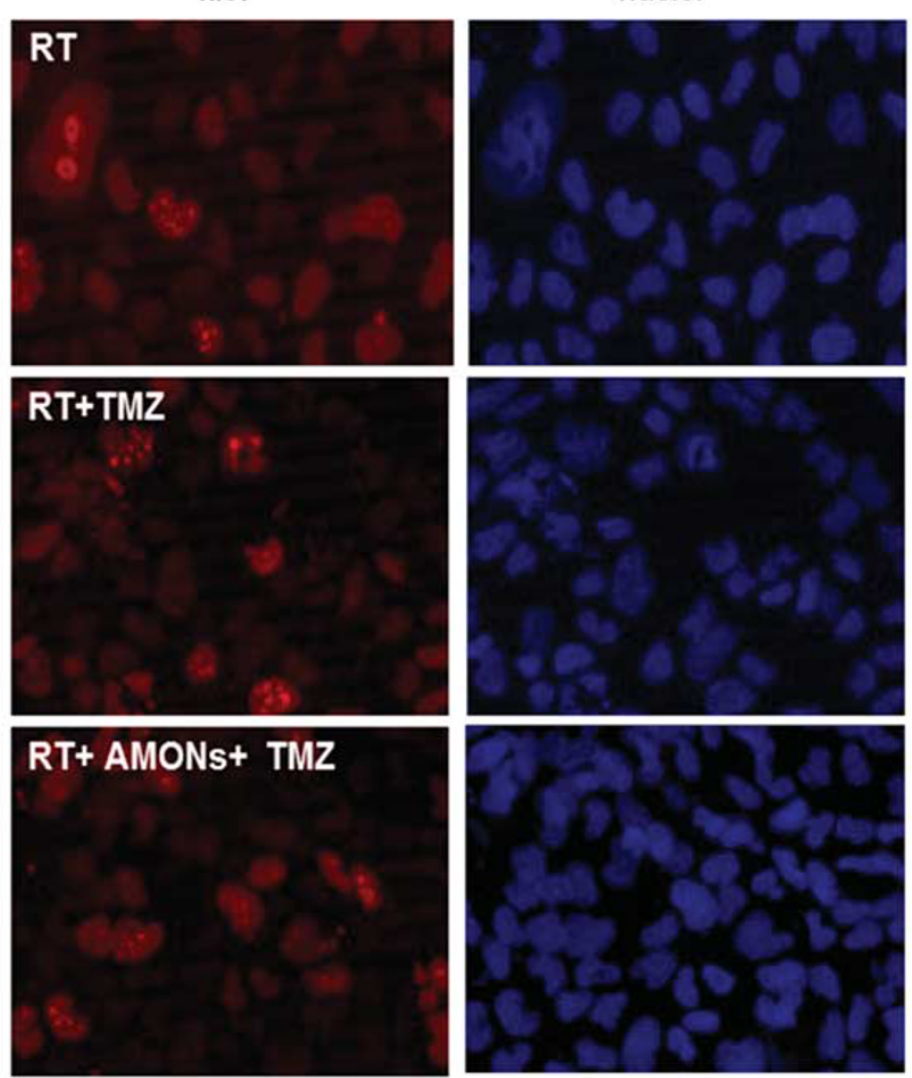

Figure 2. Downregulation of $\mathrm{O}^{6}$-methylguanine DNA methyltransferase (MGMT) by anti-MGMT oligonucleotides (AMONs) enhanced the in vitro cytotoxicity of temozolomide in T98G glioma cell lines. T98G cells were primed with $1 \mathrm{~Gy}$ followed $24 \mathrm{~h}$ later by AMONs (sequences 1,2 and 3 in 1:1:1 ratio; $5 \mu \mathrm{m}$ each). Two different doses of temozolomide (TMZ; 75 and $150 \mu \mathrm{g} \mathrm{ml}^{-1}$ ) in panels (a and b) and or $150 \mu \mathrm{g} \mathrm{ml}$ temozolomide in pnels (c-e) was added at 2 days after AMONs. Untreated controls (radiation alone and radiation plus AMONs ) were maintained. Whole-cell lysate was collected and subjected to western blotting analysis and cell viability assay was performed 1 day after temozolomide; (a) Enhanced cytotoxicity was noted in cells exposed to AMONs and temozolomide in a dose-dependent manner compared with controls. Early apoptosis of cell was indicated by phospho-p53 protein level; beta-tubulin was used as a loading control. (b) Semiquantification of western signal of phospho-p53 protein level in panel (a) of three seperately independent experiments; (c) Similar results were found when we use WST-1 cell viability assay. (d) Compared with radiation alone or radiation+TMZ, the adding of AMONs significantly decreased the percentage of ki67-positive-stained cells, suggesting that the cells were non-proliferating and cell cycle arrest at $\mathrm{G}_{0}$ stage. (e) Quantification of three random MGMT immunohistochemical fields shown in panel (d). Data are presented as mean \pm s.e.m. NS, not significant; RT, radiotherapy. Statistical significance indicated by ${ }^{*} P<0.05$. ${ }^{* *} P<0.01,{ }^{* *} P<0.001$.

hind limb tumors (Figures $3 a$ and b). ${ }^{34,35}$ We demonstrate the enhanced delivery and target downregulation only at tumor sites primed with a non-ablative dose of ionizing radiation. Pyrosequencing confirmed that the MGMT gene methylation status remained unchanged (Table 2), suggesting that the MGMT downregulation was due to posttranslational blocking rather than epigenetic silencing due to MGMT gene promoter methylation. 
a
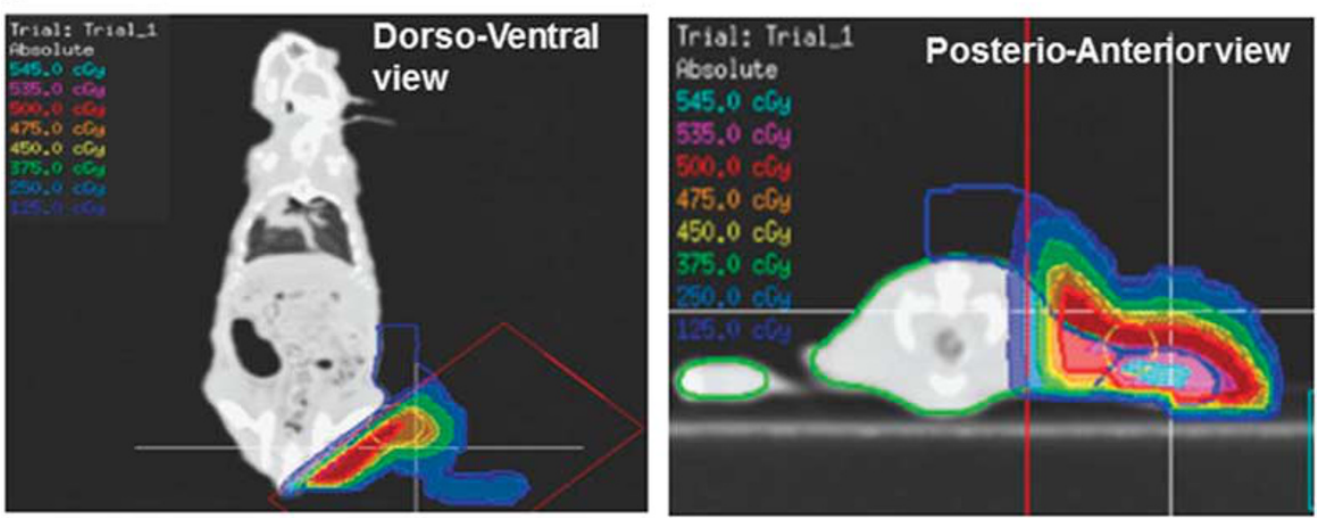

b

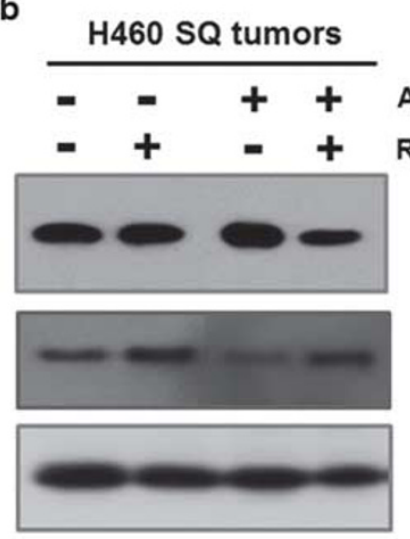

AMONs

RT (5 Gy)

MGMT

p27

tubulin

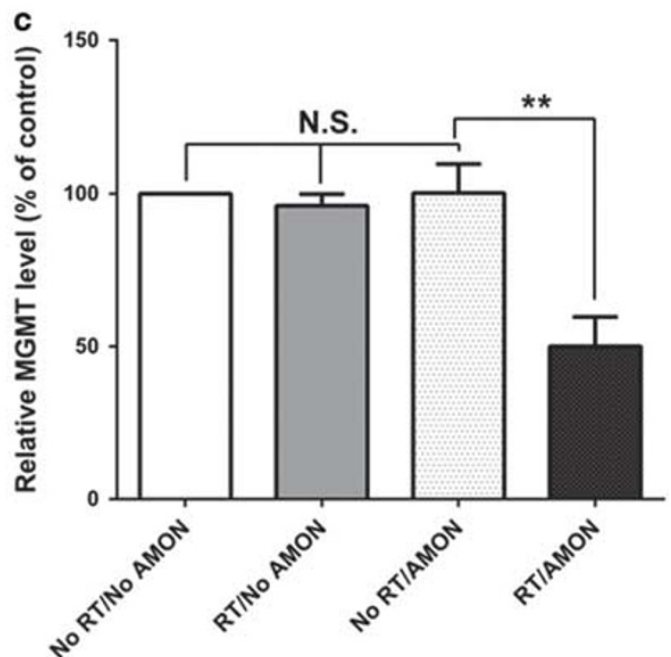

Figure 3. Radiation-guided enhanced delivery anti-MGMT oligonucleotides (AMONs) to reduce $\mathrm{O}^{6}$-methylguanine DNA methyltransferase (MGMT) expression in a $\mathrm{H} 460$ subcutaneous tumor model. Athymic nude rats were inoculated with $\mathrm{H} 460$ non-small cell lung carcinoma cells subcutaneously in both hind limbs $(n=6)$; right limb tumors received a single dose of 5 Gy radiation and left ones served as non-radiated controls. AMONs ( $15 \mu \mathrm{g} \mathrm{kg}^{-1}$; intravenous) were administered $24 \mathrm{~h}$ after radiation to half $(n=3)$ of animals and tumors were harvested 3 days later. (a) Representative CT scans with radiation field showing local delivery of radiation into right subcutaneous hindlimb tumors only; dosimetry confirmed that no radiation was delivered tumors on the contralateral hindlimb. (b) Immunoblot of MGMT and p27 of rat subcutaneous tumors; reduction in MGMT expression was found only in tumors that received both radiation and AMONs. MGMT expression was unchanged in those tumors that received AMONs or radiation only; upregulation of p27 observed in radiated (right) tumors. (c) Semiquantification of MGMT immunobloting signals from three independent experiments. NS, not significant; RT, radiotherapy.

Table 2. Summary of MGMT DNA promoter methylation percentage of $\mathrm{H} 460$ subcutaneous tumors after treatment of radiation and AMONs

\begin{tabular}{|c|c|c|c|c|}
\hline \multirow[t]{2}{*}{ Treatment $^{\mathrm{a}}$} & \multicolumn{4}{|c|}{ MGMT DNA promoter methylation ${ }^{\mathrm{b}}(\%)$} \\
\hline & Sample\#1 & Sample\#2 & Sample\#3 & Mean \pm s.d. \\
\hline No RT/no AMONs & 0 & 0 & 0 & $0 \pm 0$ \\
\hline RT/no AMONs & 1 & 1 & 0 & $0.67 \pm 0.58$ \\
\hline No RT/AMONs & 0 & 0 & 0 & $0 \pm 0$ \\
\hline RT/AMONs & 1 & 1 & 1 & $1 \pm 0$ \\
\hline \multicolumn{5}{|c|}{$\begin{array}{l}\text { Abbreviations: AMON, anti-MGMT morpholino oligonucleotide; MGMT, } \mathrm{O}^{6}- \\
\text { methylguanine DNA methyltransferase; RT, radiotherapy. }{ }^{\mathrm{a}} \mathrm{RT} \text { ( } 5 \mathrm{~Gy} \text { ) and } \\
\text { AMONs ( } 10.5 \mathrm{mg} \mathrm{kg}^{-1} \text {; intravenous). }{ }^{b} \text { Percentage of methylation of MGMT } \\
\text { DNA promoter was determined by pyrosequencing detailed in Materials } \\
\text { and Methods section. }\end{array}$} \\
\hline
\end{tabular}

Potential mechanism for radiation enhanced AMON delivery

To evaluate the mechanism by which radiation enhances AMON efficacy, the expression of key proteins involved in endocytosis pathway were assayed by western blotting from 0.5 to $48 \mathrm{~h}$ after radiation in T98G glioma cell line. Figure 4a demonstrates upregulation of caveolin 1 at 30 min after radiation, while clathrin and dynamin 2 expression peaked at $24-48 \mathrm{~h}$ after ionizing radiation. This observation was confirmed in H460 NSCLC, and caveolin 1 protein expression was upregulated and translocated from plasma membrane to cytoplasmic and perinuclear locations by immunohistochemistry at 1 and $2 \mathrm{~h}$ after radiation (Figure 4b). Figure 4c demonstrates cellular uptake of non-specific fluorescent-tagged Morpholinos in T98G cells exposed to $1 \mathrm{~Gy}$ of radiation without the use of scrape delivery of transfection agents compared with morpholino treatment alone. These findings suggest that the radiation may enhance the physiological dynamin-mediated endocytosis and may have an important role in radiation-enhanced intracellular delivery of AMON (Figure 4d).

\section{DISCUSSION}

In this study, we describe the first use of a non-ablative priming dose of ionizing radiation to guide and enhance the delivery of 
a

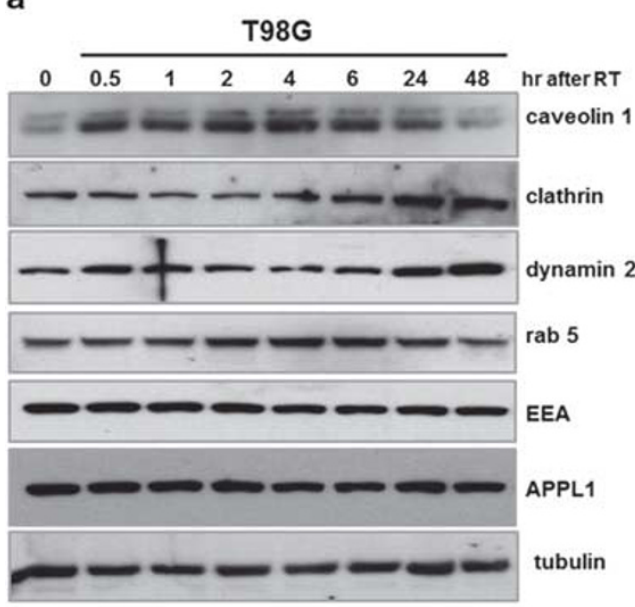

C
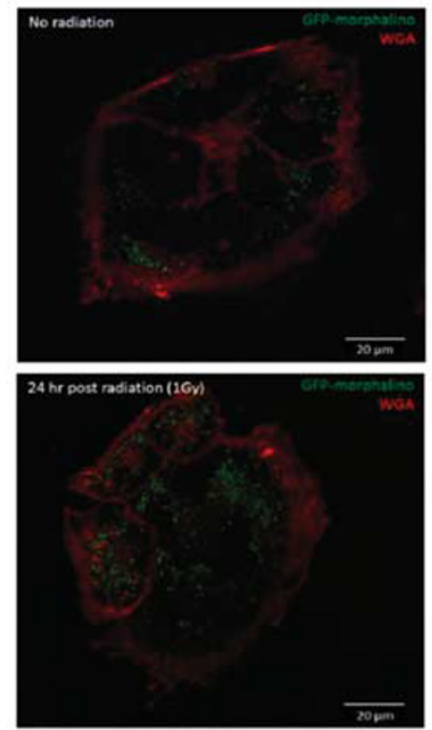

d

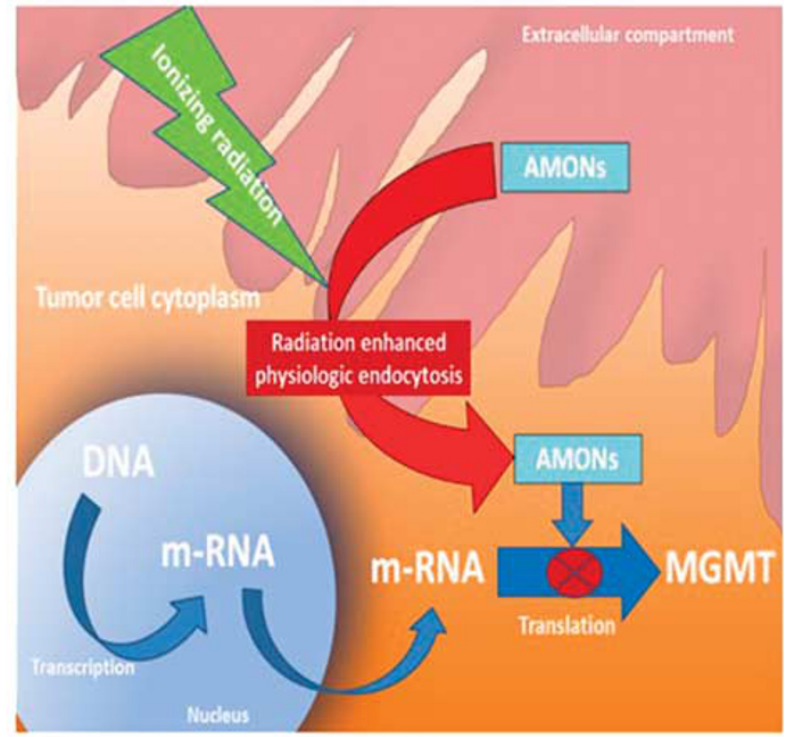

Figure 4. Radiation enhances the expression of key proteins in the physiological endocytosis pathway, possibly mediating intracellular delivery of morpholino into cancer cells. (a) Immunoblot of T98G cells showing that radiation affected the levels of endocytosis-related proteins caveolin-1, clathrin and dynamin 2, whereas others were unchanged (early endosome antigen (EEA), adaptor protein, phosphotyrosine interaction, PH domain and leucine zipper containing 1 (APPL1)); (b) Immunohistochemistry shown that radiation (6 Gy) affected caveolin-1 expression level and location in $\mathrm{H} 460$ non-small cell lung carcinoma; (c) Intracellular delivery of Green 3'-carboxyfluorescein morpholinos ( $1 \mu \mathrm{m}$; Gene Tool, LLC) in T98G cells 2 days after $1 \mathrm{~Gy}$ radiation. Red: wheat germ agglutinin (WGA) plasma membrane stain; (d) Scheme and principle of how anti-MGMT oligonucleotide (AMON) works to block $0^{6}$-methylguanine DNA methyltransferase (MGMT) protein expression. rab, Ras-related protein; RT, radiotherapy.

AMONs to downregulate MGMT expression in human tumor cells, both in vitro and in vivo in a rat xenograft tumor model. MGMT expression is a critical mechanism for resistance to CRT in solid tumors, including glioblastoma, and decreasing MGMT expression would provide significant therapeutic benefit. ${ }^{6,12,36,37}$ Various strategies to deplete MGMT have been previously reported and include the use of a dose-dense temozolomide regimen, pseudo-substrates of MGMT such as $\mathrm{O}^{6}$-Benzylguanine and $\mathrm{O}^{6}$-4-bromothenyl guanine, quinolone derivatives, S-adenosylmethionine and S-adenosylhomocysteine, and delivery of targeted short interfering RNA (siRNA) delivered in liposomes. Unfortunately, these methods face significant challenges in silencing efficacy, therapy delivery and toxic side effects. Our use of unmodified antisense morpholino oligomers for targeted protein silencing as well as the application of priming dose of non-ablative ionizing radiation to improved delivery overcomes many of these problems.

A morpholino oligomer backbone structure provides better sequence specificity than phosphorothioate-linked DNA (S-DNA) and siRNA due to (1) non-charged in physiological $\mathrm{pH}$ and hence do not bind electrostatically to proteins and are less likely to stimulate an immune response, limiting off-target effects, and (2) is resistant to the effects of nucleases. ${ }^{38,39}$ Their stability in biological systems and target specificity makes them desirable for human therapeutics. The 
recent accelerated Federal Drug Administration approval of a modified morpholino oligomer-based exon-skipping therapy demonstrated a modest yet clinically relevant restoration of dystrophin in some patients with Duchenne muscular dystrophy, indicating morpholinos as a promising tool in the clinical context. ${ }^{16,39,40}$

Although, in theory, only a very small percentage of the antisense oligonucleotides administered are required to be taken up by cells to effectively modulate target proteins, target accessibility remains a major challenge (both delivery to the tumor tissue as well as delivery across the cell membrane in the tumor). Delivery has also been an issue in previous research studies that showed promising MGMT silencing in preclinical models, but the agents did not achieve clinically relevant intratumoral distribution even with direct intratumoral injection and convection-enhanced delivery techniques. ${ }^{41-44}$ To facilitate the delivery of unmodified morpholinos across the cell membrane, the use of 'scrape loading', a vector, delivery reagents such as an endo-porter or structural modifications are required; however, these techniques have inherent limitations in the clinical setting. Structural improvements, such as the development of vivomorpholinos that are morpholino oligonucleotides covalently linked, to an octa-guanidine dendrimer delivery moiety has significantly enhanced their intracellular delivery capabilities. ${ }^{18}$ However, these structural changes that increases their molecular weight may further limit their delivery across the BBB when administered systemically. ${ }^{18}$ Intracranial delivery is also limited by specific morpholino characteristics (that is, the structure, large size and polar nature of modified antisense oligonucleotides), temperature and concentration. ${ }^{44-50}$ The current study overcomes many of these challenges by using ionizing radiation to enhance delivery of MGMT targeting particles specifically to the tumor site. This highly targeted, site-specific delivery proves the added benefit of limiting the AMON-mediated silencing to only the 'infield' site of radiation (that is, the tumor), thus decreasing toxic side effects of systemic MGMT inhibition, and being clinically applicable, as RT is standard of care in the treatment of glioblastoma. ${ }^{1,25}$ We demonstrated that the use of non-ablative radiation enhances the delivery of unmodified morpholinos both in vitro and in vivo. A single dose of AMONs was able to transiently achieve near-complete MGMT protein knockdown in vitro and $50 \%$ knockdown in vivo. Our results are comparable to the degree of in vitro MGMT knockdown previously described with $0^{6}-\mathrm{BG}$ and anti-MGMT siRNA encapsulated in cationic liposomes. ${ }^{51,52}$ The dose of priming ionizing radiation needed for optimal delivery of AMONs appears to vary among different cancer cell types (Figures $1 \mathrm{a}$, e and f). This may be due to inherent biological differences in sensitivity to radiation among various tumors. ${ }^{53-56}$

Although there is emerging interest in the use of targeted drug delivery by use of external beam radiation and there have been reports of its use to enhance the delivery and activity of targeted agents such as nanoparticles and viral vectors, the mechanism involved in radiation-mediated enhanced delivery is unclear. $^{24,42,43,57}$ Owing to the time lag noted for optimal effect after radiation (Figure 1c), we hypothesize that upregulation of the physiological endocytic pathway may be responsible for the enhanced uptake of AMONs. ${ }^{58,59}$ We found that dynamin is upregulated at $24-48 \mathrm{~h}$ after radiation while caveolin 1 is upregulated within $30 \mathrm{~min}$ and stays upregulated for up to $24 \mathrm{~h}$ after radiation exposure. Treating cells with a cell-permeable inhibitor of dynamin, dynasore, did not reduce internalization of fluorescent-tagged non-specific morpholino (data not shown), suggesting the endocytic mechanism is dynamin independent. ${ }^{60}$ lonizing radiation is known to upregulate multiple endocytosis pathways as well as increase surface antigens and receptors that could potentially facilitate endocytosis of AMONs. ${ }^{24,61}$ The cell membrane lipid bilayer is naturally impermeable to complexes $>1 \mathrm{kDa}$, alternate strategies involving the modulation of endocytosis using lipid-based drug delivery systems, nanoscale carriers and synthetic receptor targeting adapted for high affinity binding of targeting molecules and clathrin-dependent uptake are not novel and some of these are currently being evaluated in clinical trials. ${ }^{62-64}$ Radiation-mediated enhanced intracellular uptake as well as microbubble oscillation using ultrasound to deliver adenoviral vectors, plasmid DNA or siRNA have been previously described by upregulation of the physiological endocytosis pathways and our study for the first time demonstrate the use of a priming dose of non-ablative ionizing radiation-enhanced delivery of unmodified morpholino oligomers to effectively knockdown target proteins. ${ }^{24,65,66}$

The major limitation in our study is the lack of an intracranial orthotropic MGMT-expressing glioblastoma brain tumor model. This limits our ability to determine the efficacy of AMONs to cross the BBB and silence MGMT. In this context, it should be noted that RT by itself is known to increase the BBB permeability and theoretically enhance the delivery of AMONs to the tumor site, from where radiation-enhanced upregulation of physiological endocytosis will facilitate its intracellular delivery. ${ }^{67}$ MGMTexpressing glioma cell lines are less invasive and are known to be difficult to grow in animal models. ${ }^{68}$ Further, the optimal combination or ratio of sequences was not determined. Although our study suggests that good cellular uptake was noted at $24 \mathrm{~h}$ after exposure of the radiation, the effects of radiation on uptake beyond the $24 \mathrm{~h}$ point is yet to be determined. As radiation is delivered in 1.8-2 Gy daily dose fractions over 6 weeks with daily temozolomide for patients with glioblastoma, a daily IV dosing schedule may be feasible to attain sustained MGMT silencing during this period.

Our findings provide preliminary evidence to suggest that upregulation of physiological endocytosis pathway may have an important role in the enhanced AMON uptake seen in tumor cells primed with a non-ablative dose of radiation (Figure 4). Further studies are being conducted to further elucidate the mechanism of morpholino uptake in radiation-primed cells. As previously described with other morpholino oligonucleotides, MGMT knockdown achieved with a single dose of AMONs using our approach was transient. ${ }^{69}$ In the context of glioblastoma therapy, this transient MGMT knockdown is ideal for timing therapy to achieve peak knockdown with the alkylating chemotherapy, thus limiting the potential risks associated with systemic and persistent suppression of this vital DNA repair mechanism.

A single dose of AMONs (10.5 $\mathrm{mg} \mathrm{kg}^{-1}$; IV) in combination with 5 Gy local radiation achieved about 50\% MGMT protein knockdown in our animal $\mathrm{H} 460$ subcutaneous tumor model, and this dose is comparable and scalable to the $30-50 \mathrm{mg} \mathrm{kg}^{-1}$ week $^{-1}$ dose approved in treating Duchenne muscular dystrophy in clinical trial. As our goal was to determine whether targeted MGMT knockdown could be achieved, animals were killed and tumors were harvested at early time points. Significant differences in cytotoxicity were not expected at early time point, and the role of concurrent alkylating chemotherapy is an important goal for future studies. MGMT mediates chemoresistance and is frequently seen in various solid tumors, including non-small cell lung carcinoma, melanoma and breast carcinoma. Another hurdle for clinical translation of this approach to brain tumor therapy is the delivery across the BBB. ${ }^{70,71}$ Additional experiments to determine the optimal mode of delivery across the BBB with MGMTexpressing brain tumors are currently underway using a vivomorpholino backbone. ${ }^{18}$ Successful delivery across the BBB would be necessary to be effective in brain tumors, and we used 'standard' rather than vivo-morpholino backbone that has a larger molecular size for our sequences and are less likely to cross the BBB. Future experiments will evaluate additional augmented delivery techniques such as intra-arterial route with osmotic blood brain disruption, convection enhanced delivery and so on for delivery of AMONs across the BBB. 


\section{CONCLUSION}

This proof-of-concept study demonstrates that MGMT can be silenced in multiple cancer cell lines in vitro and in a subcutaneous tumor model with enhanced delivery of AMONs with a nonablated dose radiation. Our findings suggest that upregulation of key endocytosis proteins may be responsible for the enhanced delivery after radiation. This new technique for targeted delivery of unmodified morpholino oligomers has the potential for direct translation. We used MGMT as the prototype to demonstrate the proof of concept for modulating a cancer-specific protein; this technique can potentially be used to target other proteins. As RT is widely used in the management of various cancers, targeted delivery of systemically administered AMONs only to the 'field of radiation' will limit the off-target side effects. Newer molecular targets and further improvement in morpholino structure are expected to further enhance stability and efficacy of morpholinomediated protein silencing.

\section{CONFLICT OF INTEREST}

A provisional US patent application (\#62/378,780), entitled 'Morpholino oligonucleotides useful in cancer treatment' was submitted to US patent office by the authors PA, YJW and EAN. The other authors declare no conflict of interest.

\section{ACKNOWLEDGEMENTS}

This work was supported by the National Institutes of Health National Cancer Institute grants CA137488 and CA199111, a Veterans Administration Merit Review grant and the Walter S. and Lucienne Driskill Foundation, all to EAN. All morpholino oligonucleotides used were synthesized as per authors' specifications and purchased from Gene Tools, LLC, (Philomath, OR, USA) except those sequences used in experiments shown in Figure 4 that were provided free of cost. We thank $\mathrm{Dr}$ Yuangang Liu from the Department of Dermatology at OHSU for his technical support in using Leica fluorescent inverted microscope.

\section{REFERENCES}

1 Stupp R, Mason WP, van den Bent MJ, Weller M, Fisher B, Taphoorn MJ et al. Radiotherapy plus concomitant and adjuvant temozolomide for glioblastoma. N Engl J Med 2005; 352: 987-996.

2 Hegi ME, Liu L, Herman JG, Stupp R, Wick W, Weller M et al. Correlation of O6-methylguanine methyltransferase (MGMT) promoter methylation with clinical outcomes in glioblastoma and clinical strategies to modulate MGMT activity. J Clin Oncol 2008; 26: 4189-4199.

3 Zhang J, Stevens MF, Bradshaw TD. Temozolomide: mechanisms of action, repair and resistance. Curr Mol Pharmacol 2012; 5: 102-114.

4 Fu D, Calvo JA, Samson LD. Balancing repair and tolerance of DNA damage caused by alkylating agents. Nat Rev Cancer 2012; 12: 104-120.

5 Nakagawachi T, Soejima H, Urano T, Zhao W, Higashimoto K, Satoh Y et al. Silencing effect of CpG island hypermethylation and histone modifications on O6-methylguanine-DNA methyltransferase (MGMT) gene expression in human cancer. Oncogene 2003; 22: 8835-8844.

6 Sarkaria JN, Kitange GJ, James CD, Plummer R, Calvert H, Weller M et al. Mechanisms of chemoresistance to alkylating agents in malignant glioma. Clin Cancer Res 2008; 14: 2900-2908.

7 Liu L, Markowitz S, Gerson SL. Mismatch repair mutations override alkyltransferase in conferring resistance to temozolomide but not to 1,3-bis(2-chloroethyl)nitrosourea. Cancer Res 1996; 56: 5375-5379.

8 He M, Dong C, Ren R, Yuan D, Xie Y, Pan Y et al. Radiation enhances the invasiveness of irradiated and nonirradiated bystander hepatoma cells through a VEGF-MMP2 pathway initiated by p53. Radiat Res 2013; 180: 389-397.

9 Hegi ME, Diserens AC, Gorlia T, Hamou MF, de Tribolet N, Weller M et al. MGMT gene silencing and benefit from temozolomide in glioblastoma. $N$ Engl J Med 2005; 352: 997-1003.

10 Hegi ME, Diserens AC, Godard S, Dietrich PY, Regli L, Ostermann S et al. Clinical trial substantiates the predictive value of 0-6-methylguanine-DNA methyltransferase promoter methylation in glioblastoma patients treated with temozolomide. Clin Cancer Res 2004; 10: 1871-1874.

11 Tentori L, Graziani G. Recent approaches to improve the antitumor efficacy of temozolomide. Curr Med Chem 2009; 16: 245-257.
12 Ingold B, Schraml P, Heppner FL, Moch H. Homogeneous MGMT immunoreactivity correlates with an unmethylated MGMT promoter status in brain metastases of various solid tumors. PLOS ONE 2009; 4: e4775.

13 Fan $\mathrm{CH}$, Liu WL, Cao $\mathrm{H}$, Wen C, Chen L, Jiang G. O6-methylguanine DNA methyltransferase as a promising target for the treatment of temozolomideresistant gliomas. Cell Death Dis 2013; 4: e876.

14 Subbotina E, Koganti SR, Hodgson-Zingman DM, Zingman LV. Morpholino-driven gene editing: a new horizon for disease treatment and prevention. Clin Pharmacol Ther 2016; 99: 21-25.

15 Summerton J. Morpholino antisense oligomers: the case for an RNase $\mathrm{H}$-independent structural type. Biochim Biophys Acta 1999; 1489: 141-158.

16 Kinali M, Arechavala-Gomeza V, Feng L, Cirak S, Hunt D, Adkin C et al. Local restoration of dystrophin expression with the morpholino oligomer AVI-4658 in Duchenne muscular dystrophy: a single-blind, placebo-controlled, dose-escalation, proof-of-concept study. Lancet Neurol 2009; 8: 918-928.

17 Young CS, Pyle AD. Exon skipping therapy. Cell 2016; 167: 1144.

18 Morcos PA, Li Y, Jiang S. Vivo-Morpholinos: a non-peptide transporter delivers Morpholinos into a wide array of mouse tissues. Biotechniques 2008; 45: 613-614 618 passim.

$19 \mathrm{Li} \mathrm{YF}$, Morcos PA. Design and synthesis of dendritic molecular transporter that achieves efficient in vivo delivery of morpholino antisense oligo. Bioconjug Chem 2008; 19: 1464-1470.

20 Geary RS, Norris D, Yu R, Bennett CF. Pharmacokinetics, biodistribution and cell uptake of antisense oligonucleotides. Adv Drug Deliv Rev 2015; 87: 46-51.

21 Juliano RL, Ming X, Nakagawa O. Cellular uptake and intracellular trafficking of antisense and siRNA oligonucleotides. Bioconjug Chem 2012; 23: 147-157.

22 Juliano RL, Carver K. Cellular uptake and intracellular trafficking of oligonucleotides. Adv Drug Deliv Rev 2015; 87: 35-45.

23 Juliano RL, Ming X, Carver K, Laing B. Cellular uptake and intracellular trafficking of oligonucleotides: implications for oligonucleotide pharmacology. Nucleic Acid Ther 2014; 24: 101-113.

24 Qian J, Yang J, Dragovic AF, Abu-Isa E, Lawrence TS, Zhang M. lonizing radiationinduced adenovirus infection is mediated by Dynamin 2. Cancer Res 2005; 65: 5493-5497.

25 Delaney G, Jacob S, Featherstone C, Barton M. The role of radiotherapy in cancer treatment: estimating optimal utilization from a review of evidence-based clinical guidelines. Cancer 2005; 104: 1129-1137.

26 Partridge M, Vincent A, Matthews P, Puma J, Stein D, Summerton J. A simple method for delivering morpholino antisense oligos into the cytoplasm of cells. Antisense Nucleic Acid Drug Dev 1996; 6: 169-175.

27 Folkert MR, Timmerman RD. Stereotactic ablative body radiosurgery (SABR) or Stereotactic body radiation therapy (SBRT). Adv Drug Deliv Rev 2017; 109: 3-14.

$28 \mathrm{Wu}$ YJ, Neuwelt AJ, Muldoon LL, Neuwelt EA. Acetaminophen enhances cisplatinand paclitaxel-mediated cytotoxicity to SKOV3 human ovarian carcinoma. Anticancer Res 2013; 33: 2391-2400.

29 McLendon RE, Cleveland L, Pegram C, Bigner SH, Bigner DD, Friedman HS. Immunohistochemical detection of the DNA repair enzyme O6-methylguanineDNA methyltransferase in formalin-fixed, paraffin-embedded astrocytomas. Lab Invest 1998; 78: 643-644.

30 Rodriguez FJ, Thibodeau SN, Jenkins RB, Schowalter KV, Caron BL, O'Neill BP et al. MGMT immunohistochemical expression and promoter methylation in human glioblastoma. Appl Immunohistochem Mol Morphol 2008; 16: 59-65.

31 Schneider CA, Rasband WS, Eliceiri KW. NIH Image to ImageJ: 25 years of image analysis. Nat Methods 2012; 9: 671-675.

32 Dunn J, Baborie A, Alam F, Joyce K, Moxham M, Sibson R et al. Extent of MGMT promoter methylation correlates with outcome in glioblastomas given temozolomide and radiotherapy. Br J Cancer 2009; 101: 124-131.

33 Stupp R, Hegi ME, Mason WP, van den Bent MJ, Taphoorn MJ, Janzer RC et al. Effects of radiotherapy with concomitant and adjuvant temozolomide versus radiotherapy alone on survival in glioblastoma in a randomised phase III study: 5-year analysis of the EORTC-NCIC trial. Lancet Oncol 2009; 10: 459-466.

34 Chaudhry MA. Biomarkers for human radiation exposure. J Biomed Sci 2008; 15: 557-563.

35 Kang CM, Park KP, Song JE, Jeoung DI, Cho CK, Kim TH et al. Possible biomarkers for ionizing radiation exposure in human peripheral blood lymphocytes. Radiat Res 2003; 159: 312-319.

36 Gerson SL. MGMT: its role in cancer aetiology and cancer therapeutics. Nat Rev Cancer 2004; 4: 296-307.

37 Kitange GJ, Carlson BL, Schroeder MA, Grogan PT, Lamont JD, Decker PA et al. Induction of MGMT expression is associated with temozolomide resistance in glioblastoma xenografts. Neuro Oncol 2009; 11: 281-291.

38 Summerton JE. Morpholino, siRNA, and S-DNA compared: impact of structure and mechanism of action on off-target effects and sequence specificity. Curr Top Med Chem 2007; 7: 651-660. 
39 Kole R, Krieg AM. Exon skipping therapy for Duchenne muscular dystrophy. Adv Drug Deliv Rev 2015; 87: 104-107.

40 Aartsma-Rus A, Krieg AM. FDA approves eteplirsen for duchenne muscular dystrophy: the next chapter in the eteplirsen saga. Nucleic Acid Ther 2017; 27: 1-3.

41 Tsujiuchi T, Natsume A, Motomura K, Kondo G, Ranjit M, Hachisu R et al. Preclinical evaluation of an $\mathrm{O}(6)$-methylguanine-DNA methyltransferase-siRNA/liposome complex administered by convection-enhanced delivery to rat and porcine brains. Am J Transl Res 2014; 6: 169-178.

42 Wang J, Lu Z, Wientjes MG, Au JL. Delivery of siRNA therapeutics: barriers and carriers. AAPS J 2010; 12: 492-503.

43 Viel T, Boisgard R, Kuhnast B, Jego B, Siquier-Pernet K, Hinnen F et al. Molecular imaging study on in vivo distribution and pharmacokinetics of modified small interfering RNAs (siRNAs). Oligonucleotides 2008; 18: 201-212.

44 Akhtar S, Juliano RL. Cellular uptake and intracellular fate of antisense oligonucleotides. Trends Cell Biol 1992; 2: 139-144.

45 Loke SL, Stein CA, Zhang XH, Mori K, Nakanishi M, Subasinghe C et al. Characterization of oligonucleotide transport into living cells. Proc Natl Acad Sci USA 1989; 86: 3474-3478.

46 Yakubov LA, Deeva EA, Zarytova VF, Ivanova EM, Ryte AS, Yurchenko LV et al. Mechanism of oligonucleotide uptake by cells: involvement of specific receptors? Proc Natl Acad Sci USA 1989; 86: 6454-6458.

47 Beltinger C, Saragovi HU, Smith RM, LeSauteur L, Shah N, DeDionisio L et al. Binding, uptake, and intracellular trafficking of phosphorothioate-modified oligodeoxynucleotides. J Clin Invest 1995; 95: 1814-1823.

48 Roth CM. Molecular and cellular barriers limiting the effectiveness of antisense oligonucleotides. Biophys J 2005; 89: 2286-2295.

49 Vlassov VV, Balakireva LA, Yakubov LA. Transport of oligonucleotides across natural and model membranes. Biochim Biophys Acta 1994; 1197: 95-108.

50 Dias N, Stein CA. Antisense oligonucleotides: basic concepts and mechanisms. Mol Cancer Ther 2002; 1: 347-355.

51 Kato T, Natsume A, Toda H, Iwamizu H, Sugita T, Hachisu R et al. Efficient delivery of liposome-mediated MGMT-siRNA reinforces the cytotoxity of temozolomide in GBM-initiating cells. Gene Therapy 2010; 17: 1363-1371.

52 Konduri SD, Ticku J, Bobustuc GC, Sutphin RM, Colon J, Isley B et al. Blockade of MGMT expression by $\mathrm{O} 6$ benzyl guanine leads to inhibition of pancreatic cancer growth and induction of apoptosis. Clin Cancer Res 2009; 15: 6087-6095.

53 Theys J, Yahyanejad S, Habets R, Span P, Dubois L, Paesmans K et al. High NOTCH activity induces radiation resistance in non small cell lung cancer. Radiother Oncol 2013; 108: 440-445.

54 Eichler AF, Loeffler JS. Multidisciplinary management of brain metastases. Oncologist 2007; 12: 884-898.

55 Shaw E, Scott C, Souhami L, Dinapoli R, Kline R, Loeffler J et al. Single dose radiosurgical treatment of recurrent previously irradiated primary brain tumors and brain metastases: final report of RTOG protocol 90-05. Int J Radiat Oncol Biol Phys 2000; 47: 291-298.

56 Bleehen NM, Stenning SP. A Medical Research Council trial of two radiotherapy doses in the treatment of grades 3 and 4 astrocytoma. The Medical Research Council Brain Tumour Working Party. Br J Cancer 1991; 64: 769-774.
57 Hariri G, Yan H, Wang H, Han Z, Hallahan DE. Radiation-guided drug delivery to mouse models of lung cancer. Clin Cancer Res 2010; 16: 4968-4977.

58 Rewatkar PV, Parton RG, Parekh HS, Parat MO. Are caveolae a cellular entry route for non-viral therapeutic delivery systems? Adv Drug Deliv Rev 2015; 91: 92-108.

59 Mayor S, Pagano RE. Pathways of clathrin-independent endocytosis. Nat Rev Mol Cell Biol 2007; 8: 603-612.

60 Macia E, Ehrlich M, Massol R, Boucrot E, Brunner C, Kirchhausen T. Dynasore, a cell-permeable inhibitor of dynamin. Dev Cell 2006; 10: 839-850.

61 Kaliberov SA, Kaliberova LN, Yan H, Kapoor V, Hallahan DE. Retargeted adenoviruses for radiation-guided gene delivery. Cancer Gene Ther 2016; 23: 303-314.

62 Yingchoncharoen P, Kalinowski DS, Richardson DR. Lipid-based drug delivery systems in cancer therapy: what is available and what is yet to come. Pharmacol Rev 2016; 68: 701-787.

63 Bareford LM, Swaan PW. Endocytic mechanisms for targeted drug delivery. Adv Drug Deliv Rev 2007; 59: 748-758.

64 Hymel D, Peterson BR. Synthetic cell surface receptors for delivery of therapeutics and probes. Adv Drug Deliv Rev 2012; 64: 797-810.

65 Egami T, Ohuchida K, Mizumoto K, Onimaru M, Toma H, Nishio S et al. Radiation enhances adenoviral gene therapy in pancreatic cancer via activation of cytomegalovirus promoter and increased adenovirus uptake. Clin Cancer Res 2008; 14: 1859-1867.

66 Delalande A, Leduc C, Midoux P, Postema M, Pichon C. Efficient gene delivery by sonoporation is associated with microbubble entry into cells and the clathrindependent endocytosis pathway. Ultrasound Med Biol 2015; 41: 1913-1926.

67 McGuire HH Jr, Horsley JS 3rd, Salter DR, Sobel M. Measuring and managing quality of surgery. Statistical vs incidental approaches. Arch Surg 1992; 127: 733-737 discussion 738

68 Chahal M, Abdulkarim B, Xu Y, Guiot MC, Easaw JC, Stifani N et al. O6-Methylguanine-DNA methyltransferase is a novel negative effector of invasion in glioblastoma multiforme. Mol Cancer Ther 2012; 11: 2440-2450.

69 Ferguson DP, Schmitt EE, Lightfoot JT. Vivo-morpholinos induced transient knockdown of physical activity related proteins. PLOS ONE 2013; 8: e61472.

70 Neuwelt E, Ambady P, Muldoon L, McConnell H, Doolittle N. Outwitting the blood-brain barrier. Oncology (Williston Park) 2016; 30: 963, 966-967.

71 Puhalla S, Elmquist W, Freyer D, Kleinberg L, Adkins C, Lockman P et al. Unsanctifying the sanctuary: challenges and opportunities with brain metastases. Neuro Oncol 2015; 17: 639-651.

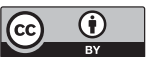

This work is licensed under a Creative Commons Attribution 4.0 International License. The images or other third party material in this article are included in the article's Creative Commons license, unless indicated otherwise in the credit line; if the material is not included under the Creative Commons license, users will need to obtain permission from the license holder to reproduce the material. To view a copy of this license, visit http://creativecommons.org/licenses/ by/4.0/

c The Author(s) 2017

Supplementary Information accompanies the paper on Cancer Gene Therapy website (http://www.nature.com/cgt) 